\title{
COMPARISON BETWEEN RANKING METHOD AND THE ANALYTIC HIERARCHY PROCESS: IN THE APPLICATION TO PROGRAM POLICY ANALYSIS
}

\author{
Yuji Sato \\ Department of Policy Science \\ Matsusaka University \\ 1846, Kubo, Matsusaka, Mie, 515-8511 - Japan \\ ysatoh@matsusaka-u.ac.jp
}

\begin{abstract}
The purpose of this paper is to examine the relative effectiveness of a ranking method for measuring human perception. Specifically, the answers to two different sets of a pair of questions on a particular issue are compared: one set is formatted using a ranking format; the other set is formatted using the Analytic Hierarchy Process (AHP) format.

Questionnaire design for survey research, such as public opinion polls, presents one of the most challenging and controversial issues for survey researchers in terms of accuracy in measuring respondents' perceptions. Consequently, many ways of asking questions have been proposed and much discussion has been generated.

One rating scale - called a Feeling Thermometer - was developed by a group of researchers at the University of Michigan. The scale ranges from 0 , the coldest feeling toward alternatives, to 100 , the hottest, with 50, being neutral. In surveys, respondents express their perceptions by indicating their "temperature" for each alternative for a given question. Although this method helps respondents precisely clarify their judgments for each alternative, the relative judgments across alternatives is not captured.
\end{abstract}

A more traditional method for measuring respondents' perceptions is the multiple-choice question format, which has been thought to be highly suitable for questionnaire formatting because respondents find them easy to answer and they allow researchers to easily identify the main concerns of the respondents. This method, however, has been shown in the author's previous research not to be effective in terms of accuracy in reflecting human perceptions upon a result, particularly for questions concerning "delicate" issues.

Another scale that has been developed is the ranking scale used by Ronald Inglehart and Paul Abramson in their World Values Survey. This method asks respondents to rank all given alternatives in a question, from the most preferred to the least, thus allowing researchers to identify a respondent's preference order for all alternatives.

One possible option for formatting questionnaire is to apply the AHP, a popular method for decision-making developed by Thomas L. Saaty. Data from a decision-maker's pair-wise comparisons are aggregated, and the degree of importance of each alternative is quantified in the AHP. This quantification identifies not only the most important alternative but also the preference for all alternatives for each decision maker. Using the AHP to analyze a decision-making process, therefore, results in a precise clarification of preferences for alternatives.

In this paper, we compare the ranking method and the AHP method in terms of appropriateness for representing each respondent's perception. Two different sets of a pair of questions on a particular issue are used to compare the two methods. One concerns an abstract issue - the philosophy of refining 
governmental program policy; the other relates to a concrete issue - the governmental projects with high priority. In the comparisons, the respondents were executive staff members of the prefectural government who have authority for final budget decisions.

The paper focuses on the difference of preference orders for all alternatives between the ranking format and the AHP format. Two findings are: (1) for the abstract issue, the ranking format and the AHP format yielded similar aggregated rankings of alternatives, and (2) for the concrete issue, the results from the AHP format were superior to those generated by the ranking format since the AHP results coincided almost exactly with the annual change in the actual implementation of the budget. 